\title{
Jets and Vector Bosons in Heavy lon Collisions
}

\author{
Begoña de la Cruz on behalf of CMS Collaboration ${ }^{1, a}$ \\ ${ }^{1}$ CIEMAT, Madrid
}

\begin{abstract}
This paper reviews experimental results on jets and electroweak boson (photon, $\mathrm{W}$ and $\mathrm{Z}$ ) production in heavy-ion collisions, from the CMS and ATLAS detectors, using data collected during $2011 \mathrm{PbPb}$ run and pp data collected at an equivalent energy. By comparing the two collision systems, the energy loss of the partons propagating through the medium produced in $\mathrm{PbPb}$ collisions can be studied. Its characterization is done using dijet events and isolated photon-jet pairs. Since the electroweak gauge bosons do not participate in the strong interaction, and are thus unmodified by the nuclear medium, they serve as clean probes of the initial state in the collision.
\end{abstract}

\section{Introduction}

In heavy nuclei collisions at very high energies, quarks and gluons, which are confined into colourless hadrons, become free, having the capability to form a high density colour deconfined state of strongly interacting matter, called Quark-Gluon Plasma (QGP). Lattice QCD calculations predict the existance of a phase transition for reaching the QGP, given the high temperatures and energy densities reached in the heavy ion collisions. The high-momentum partons formed in the early stages of the collisions, traverse the hot medium, losing energy as they interact with other constituents via radiative or collisional processes. These energetic partons give rise to final particles which are sensitive to the electroweak interaction, like $\mathrm{W}, \mathrm{Z}$ and photons, or to the strong force, like quarks and gluons which reach the detectors as jets of particles, after hadronization. Both kind of probes, jets and bosons, provide very valuable information on the energy loss mechanism and the partonic medium characteristics and structure.

One of the experimental programs addressing nowadays heavy ion collisions takes place at the LHC (Large Hadron Collider) at CERN (Geneva) and during the three years of high-performant operation of this accelerator, several samples of $\mathrm{PbPb}, \mathrm{pPb}$ and $\mathrm{pp}$ collision data have been gathered by ATLAS, ALICE and CMS experiments ${ }^{1}$.

In the following, results obtained by CMS and ATLAS on the subject of jets and vector boson production in heavy ion collisions are presented. For that purpose, two sets of $\mathrm{PbPb}$ data at $\sqrt{s}=2.76 \mathrm{TeV}$ per interacting nucleon pair have been analysed, one comprising an integrated luminosity of around $7 \mu \mathrm{b}^{-1}$ (2010 PbPb Run) and another one with $150 \mu \mathrm{b}^{-1}$ (2011 $\mathrm{PbPb}$ Run). For reference purposes, two sets of pp data at the same centre-of-mass en-

\footnotetext{
a e-mail: begona.delacruz@ciemat.es

${ }^{1} \mathrm{LHCb}$ experiment has also collected $\mathrm{pp}$ and $\mathrm{pPb}$ data samples.
}

ergy $\left(\mathcal{L}=230 \mathrm{nb}^{-1}\right.$ and $\mathcal{L}=5 \mathrm{pb}^{-1}$, collected in 2011 and 2013 respectively) were also studied. Finally, preliminary results on a new set of data with $\mathrm{pPb}$ collisions at $\sqrt{s_{\mathrm{NN}}}=5 \mathrm{TeV}$ corresponding to an integrated luminosity of $31 \mathrm{nb}^{-1}$, recorded at the beginning of 2013 , were presented.

\section{Jet production}

Given the high center-of-mass energies achieved in heavy ion collisions at the LHC, jets of high $\mathrm{p}_{\mathrm{T}}$ are produced at higher rates than in prior experiments and thus are useful probes to test properties of the partonic medium created.

A very important characteristic of jet production was established already at RHIC (Relativistic Heavy Ion Collider), namely the jet-quenching, the suppression of high$\mathrm{p}_{\mathrm{T}}$ particles. This effect has been confirmed to be present in $\mathrm{PbPb}$ collisions at the LHC, and has been studied in the dijet event production, quantifying it by means of the jet asymmetry, $\mathrm{A}_{\mathrm{J}}=\frac{p_{\mathrm{T}}^{\mathrm{J1}}-p_{\mathrm{T}}^{\mathrm{J} 2}}{p_{\mathrm{T}}^{\mathrm{J1}}+p_{\mathrm{T}}^{p^{2}}}$, where $\mathrm{J} 1$ and $\mathrm{J} 2$ refer to the leading and next-to-leading $\mathrm{p}_{\mathrm{T}}$ jets in each event. Jets are reconstructed using an anti- $\mathrm{k}_{\mathrm{T}}$ algorithm, with radius $\mathrm{R}=0.3$ 0.4 , and samples of events containing a jet of $\mathrm{p}_{\mathrm{T}}>100$ $\mathrm{GeV} / c\left(\right.$ ATLAS[1]) and $\mathrm{p}_{\mathrm{T}}>120 \mathrm{GeV} / c(\mathrm{CMS}[2])$ are considered. A value of $A_{J} \sim 0$ implies a balanced dijet configuration, while $A_{J}>>0$ indicates a suppression of the $\mathrm{p}_{\mathrm{T}}$ of the second jet relative to the first one. This quantity is studied for different collision centralities (reflecting the geometrical overlap of the colliding nuclei) observing a clear shift of the mean value of the distribution to higher values of $\mathrm{A}_{J}$ for very central $\mathrm{PbPb}$ collisions (large overlap of the incident nuclei and thus great number of participating nucleons), compared to the same distribution in pp data at the same centre-of-mass energy and to predictions from dijet production by PYTHIA embedded in $\mathrm{PbPb}$ events simulated with HIJING (ATLAS) and experimental minimum bias $\mathrm{PbPb}$ data (CMS). 
Despite the large asymmetries, the distribution of the difference of the jets azimuthal angles is highly peaked towards a back-to-back configuration, almost independently of the centrality of the nuclear collision and in good agreement with the corresponding distribution from pp data and from simulation expectations.

The momentum lost by the subleading jet is transferred to the medium. The momentum balance in the event is restored when all charged particles with momenta as low as $0.5 \mathrm{GeV} / c$ are considered. Their spatial distribution is such, that high $\mathrm{p}_{\mathrm{T}}$ particles are closer to the leading jet axis in the transverse plane, while the low $\mathrm{p}_{\mathrm{T}}$ particles which compensate and balance the event are produced at much bigger angles relative to the jet axis, as measured by $\mathrm{CMS}[2]$. This results in wider jets in central $\mathrm{PbPb}$ collisions, compared to $\mathrm{pp}$ interactions, as there is an enhancement in the contribution of soft $\mathrm{p}_{\mathrm{T}}\left(\mathrm{p}_{\mathrm{T}} \leq 4 \mathrm{GeV} / c\right)$ particles to the emerging jet, at large angles respect to its axis. Quantitatively, fragmentation functions describing the hadronization of quarks and gluons in jets, have been measured by both experiments[3][4], using the variable $\mathrm{z}=\mathrm{p}_{\|}^{\mathrm{tr}} / \mathrm{p}^{\text {jet }}$ which relates the momentum component of charged tracks within a jet cone to its momentum axis, $\mathrm{p}^{\text {jet }}$. Figure 1 shows the distribution of $\xi=\ln (1 / z)$ measured by CMS for different ranges of the collision centrality, referred to the same quantity computed in pp interactions at the same centre-of-mass energy. The ratio is compatible with unity for peripheral collisions (left pannel), while it significantly deviates from one at low values of $\mathrm{Z}$ (large angles relative to the jet axis) for central collisions. ATLAS performs the measurement in terms of the ratio of central to peripheral collisions, reaching similar conclusions.

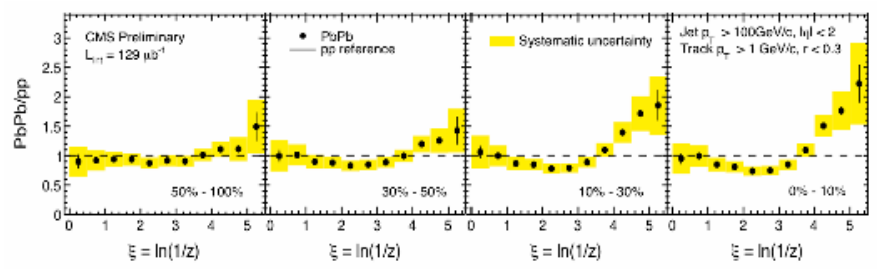

Figure 1. Ratio of fragmentation functions in $\mathrm{PbPb}$ collisions, obtained by CMS, for jets with $\mathrm{p}_{T}>100 \mathrm{GeV} / c$ and tracks with $\mathrm{p}_{T}>1 \mathrm{GeV} / c$, for four regions in centrality, relative to their $\mathrm{pp}$ reference.

Besides the reduction of transverse momenta of jets in relativistic heavy ion collisions, the inclusive yield of these high- $\mathrm{p}_{\mathrm{T}}$ jets is also affected. This is quantified through the nuclear modification factor, $\mathrm{R}_{A A}$, defined as the ratio of the inclusive jet yield in $\mathrm{PbPb}$ and in pp collisions, normalised by the number of binary nucleon-nucleon collisions.

Figure 2 presents this quantity as a function of the number of participants, as measured by CMS[5], showing a factor of two suppression in high- $\mathrm{p}_{\mathrm{T}}$ jet yields in central $\mathrm{PbPb}$ collisions, relative to those measured in pp interactions. This suppression is almost independent of the jet $\mathrm{p}_{\mathrm{T}}$ and cone radius, as the ATLAS[6] results on Figure 3 shows, in the ratio of yields in central and peripheral collisions.

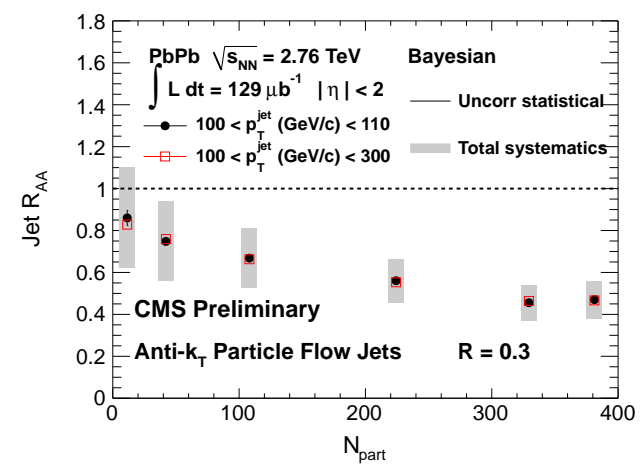

Figure 2. Jet $\mathrm{R}_{\mathrm{AA}}$ as a function of $\mathrm{N}_{\text {part }}$ for $100<$ jet $\mathrm{p}_{\mathrm{T}}<110$ $\mathrm{GeV} / \mathrm{c}$ (closed circles) and $100<$ jet $\mathrm{p}_{\mathrm{T}}<300 \mathrm{GeV} / \mathrm{c}$ (open boxes) from CMS.

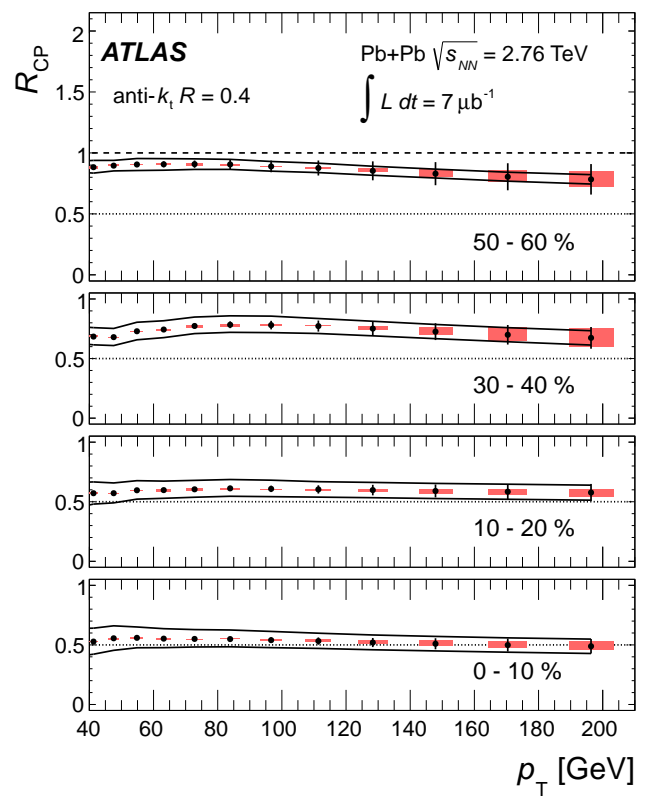

Figure 3. Jet $\mathrm{R}_{\mathrm{CP}}$ (relative yield in central to peripheral collisions) as a function of jet $\mathrm{p}_{T}$.

CMS and ATLAS have also performed a first measurement of the fraction of the inclusive jet yields coming from b-quarks in $\mathrm{PbPb}$ data. ATLAS[7] exploits for that purpose the $\mathrm{p}_{\mathrm{T}}$ spectrum of single muons coming in jets, measured in various subdetectors, within the transverse momentum range $4<\mathrm{p}_{\mathrm{T}}<14 \mathrm{GeV} / c$.

CMS[8] uses the relatively long b-quark lifetime, which gives rise to secondary vertices in the decay of Blike mesons. This way of tagging the presence of a b-quark in a jet is quite challenging, given the high multiplicity environment. Fitting the reconstructed mass of the particles coming from the secondary vertex, with a b-quark component plus a c- and light-quark ones, the fraction of b-jets 
relative to the total inclusive one is extracted, for jets with $\mathrm{p}_{\mathrm{T}}>100 \mathrm{GeV} / c$. Within uncertainties, and related to the fraction measured in pp collisions, the $\mathrm{PbPb}$ data favours a scenario where b-jets suffer energy losses similar to those in the inclusive jet sample.

Finally, only a few months ago, pPb collisions data were collected, at a centre-of-mass energy of $5 \mathrm{TeV}$. First studies of dijet events by CMS[9] indicate that there is no evident $\mathrm{p}_{\mathrm{T}}$ imbalance in jets in $\mathrm{pPb}$ collisions, consistent with results from the $\mathrm{pp}$ reference sample and with $\mathrm{pPb}$ HIJING simulations.

\section{Vector Boson production}

\subsection{Isolated photons}

Photons coming directly from the hard scattering of incident nucleons are usually highly energetic and produced isolated from other particles. This is not the case for photons from decays of neutral mesons produced inside a jet and those from parton fragmentation, and thus, surrounded by significant hadronic activity from other parton fragments. Isolation provides a good handle to suppress the fragmentation photon component in the data sample, while removing a very small contribution of direct photons. Still, the hard scattering is superimposed on top of other multiple parton-parton scatterings (underlying event) occurring simultaneously and which must be subtracted, prior to applying the isolation criteria during the event selection.

Despite the challenge in disentagling both photon contributions, CMS[10] and ATLAS[11] have succeeded in selecting a sample of isolated photons, dominated by photons originating from quark-gluon Compton scattering, with only a small portion of photons from decays and fragmentation processes, which fulfill the isolation criteria.

Figure 4 shows the isolated photon transverse momentum distribution measured by ATLAS in $\mathrm{PbPb}$ data for four different regions in centrality ( [0-10\%], [10-20\%], [20$40 \%$ ] and [40-80\%]) and evaluated in the central region in photon pseudorapidity $\left|\eta^{\gamma}\right| \leq 1.4$. The corresponding cross section obtained in pp data, at the same centre-ofmass energy, measured by CMS, is also included. The photon $\mathrm{p}_{\mathrm{T}}$ distributions from $\mathrm{PbPb}$ data, scaled by ${ }^{2} \mathrm{~T}_{A A}$, agree quite well with those from $\mathrm{pp}$ collisions and with NLO JETPHOX calculations.

From this comparison, nuclear modification factors $\mathrm{R}_{\mathrm{AA}}$ are derived. This is done for different centrality regions, as a function of photon $\mathrm{p}_{\mathrm{T}}$. Figure 5 shows the $\mathrm{R}_{\mathrm{AA}}$ dependence with the number of participants, for different ranges of photon $\mathrm{E}_{\mathrm{T}}$, as measured by CMS. The error bars account for the statistical uncertainty and the coloured-filled rectangles for the systematic ones, which come mainly from the background template modelling and the photon energy scale, both in the $\mathrm{PbPb}$ and in the $\mathrm{pp}$ data. The $\mathrm{R}_{\mathrm{AA}}$ values are compatible with unity and, thus, confirm the scaling of the isolated photon production with $\mathrm{N}_{\text {coll }}$, establishing the isolated photon sample as a baseline for further analyses, as will be shown later.

\footnotetext{
${ }^{2} \mathrm{~T}_{\mathrm{AA}}$ is the number of elementary binary nucleon-nucleon (NN) collisions, $\mathrm{N}_{\text {coll }}$, divided by the elementary $\mathrm{NN}$ cross section.
}

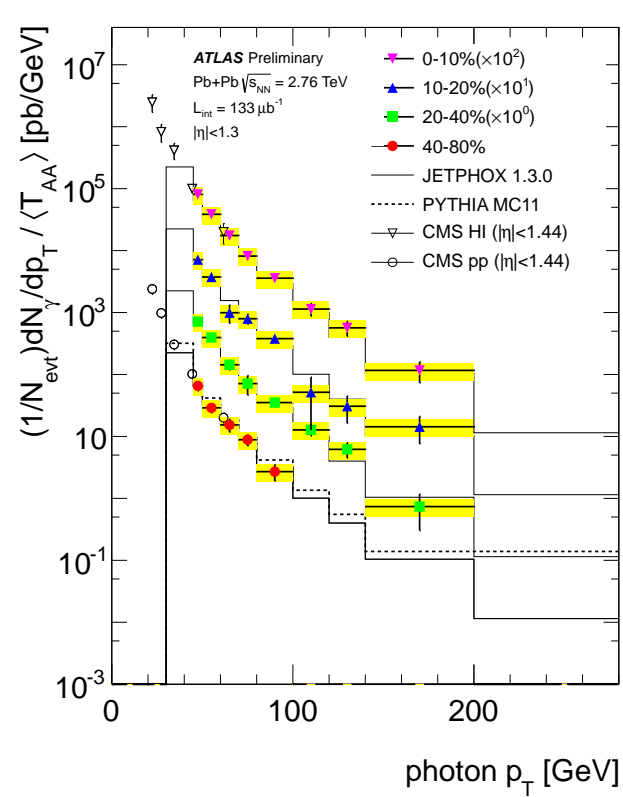

Figure 4. Isolated photon spectra as a function of the photon $\mathrm{p}_{\mathrm{T}}$ for several centrality regions in $\mathrm{PbPb}$ collisions (scaled by $\mathrm{T}_{\mathrm{AA}}$ ) and pp collisions, scaled by arbitrary factors (for better visualization). The results are compared to NLO JETPHOX calculations.

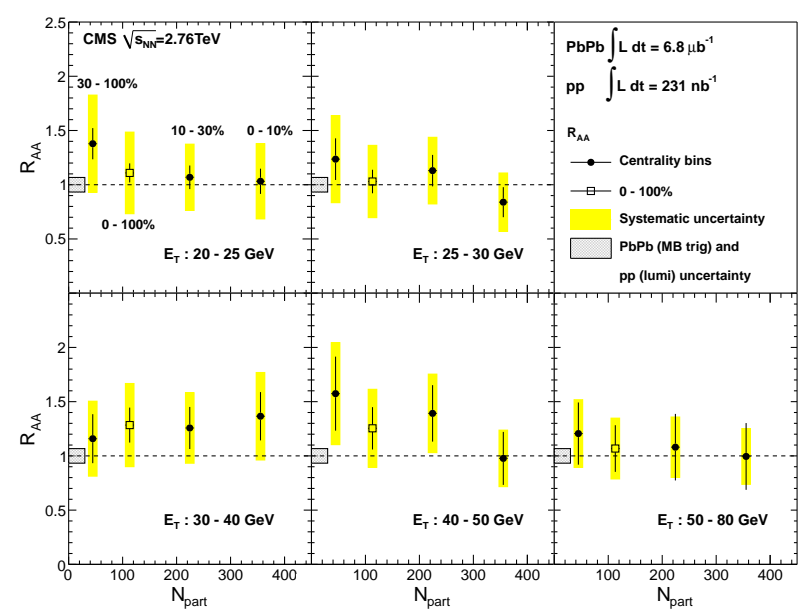

Figure 5. Nuclear modification factors, $\mathrm{R}_{A A}$, as a function of $\mathrm{PbPb}$ centrality (given by the number of participant nucleons, $\mathrm{N}_{\text {part }}$ ) for five different photon $\mathrm{E}_{\mathrm{T}}$ intervals.

\section{$3.2 \mathrm{Z}$ bosons}

The $\mathrm{Z}$ and $\mathrm{W}$ bosons have been detected and measured at the LHC for the first time in heavy ion collisions, despite their very low production cross section.

In the case of the $\mathrm{Z}$ bosons, the decay channels studied in both ATLAS[12] and CMS[13] are Z $\rightarrow \mu^{+} \mu^{-}$and $\mathrm{Z} \rightarrow e^{+} e^{-}$. The selection of events in these channels exploits their quite clean signature: two oppositely charged high-transverse-momenta leptons $\left(\mathrm{p}_{\mathrm{T}}^{\mu}>10-20 \mathrm{GeV} / \mathrm{c}\right)$, yielding very clear dilepton invariant mass peaks, with 
an almost negligible background. Figure 6 presents the dimuon (left) and dielectron (right) invariant mass distributions, based on an integrated luminosity $\mathcal{L}=0.15 \mathrm{nb}^{-1}$ of $\mathrm{PbPb}$ collisions data, measured by ATLAS.

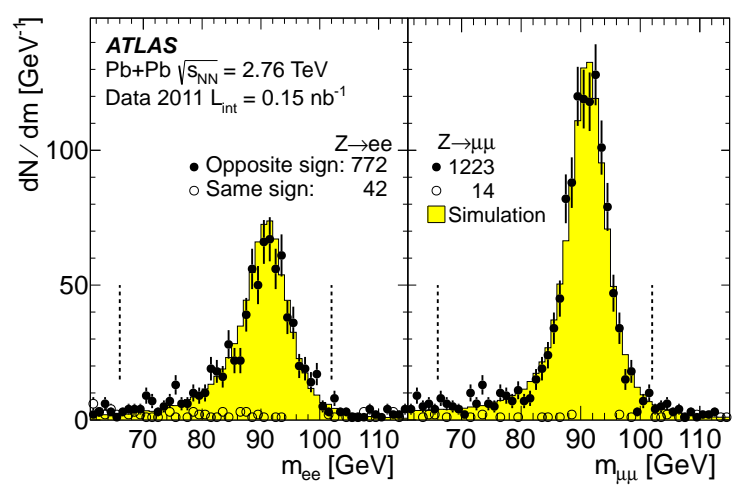

Figure 6. Dilepton invariant mass spectra in $\mathrm{PbPb}$ collisions: dielectrons (left plot), dimuons (right plot). Full circles are opposite-sign dileptons, while empty circles show same-sign dilepton candidates. The solid histogram shows the expectations from the simulated events $\left(\mathrm{pp} \rightarrow \mathrm{Z} \rightarrow l^{+} l^{-}\right.$) embedded in $\mathrm{PbPb}$ HIJING events. Vertical lines correspond to statistical uncertainties.

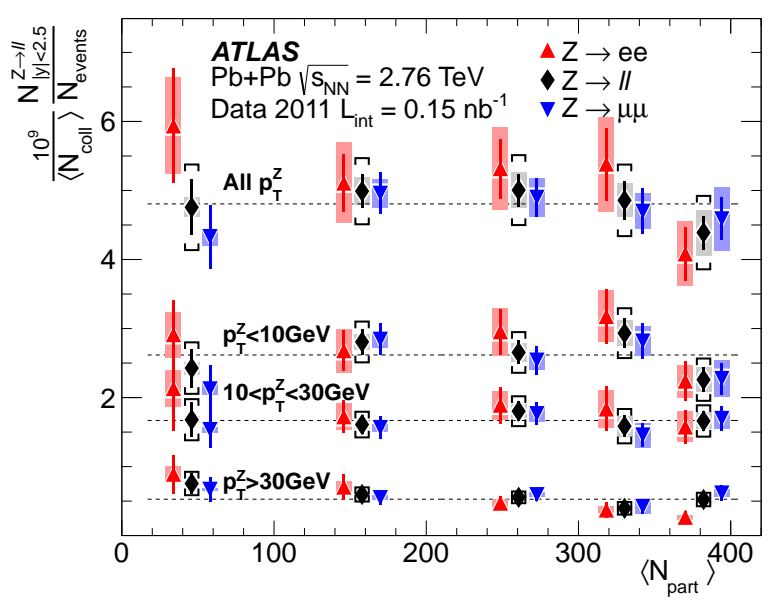

Figure 7. Centrality dependence (as a function of Npart) of $\mathrm{Z}$ boson yields normalised to Ncoll in $\mathrm{PbPb}$ collisions, measured by ATLAS, for ee (upward pointing triangles) and $\mu \mu$ (downward pointing triangles) channels, and their weighted average (diamonds). Vertical lines (bands) correspond to statistical (systematic) uncertainties. The dashed lines show the result of the fit to a constant value.

Several kinematic distributions have been studied with the samples of $Z$ candidates, like the $Z$ rapidity and $p_{T}$, and have been compared to Powheg (CMS) and PYTHIA normalised to NNLO cross section (ATLAS) predictions for $\mathrm{pp} \rightarrow \mathrm{Z} \rightarrow l^{+} l^{-}$, scaled with the number of binary collisions. The experimental distributions are consistent with both theoretical predictions.
Examining $\mathrm{PbPb}$ collisions of different centrality, no dependence of the production of $\mathrm{Z}$ bosons on the number of participants is observed (see Figure 7), shown inclusively for the dimuon and dielectron channels, and also in three different $\mathrm{p}_{\mathrm{T}}(Z)$ ranges. The same behaviour is observed relative to the boson rapidity. These results validate the $\mathrm{Z}$ bosons as a reference particle unaffected by finalstate interactions in the strongly interacting dense system produced in $\mathrm{Pb}-\mathrm{Pb}$ collisions at the $\mathrm{LHC}$.

\subsection{W bosons}

The production of $\mathrm{W}$ bosons in $\mathrm{PbPb}$ collisions has been measured at the LHC in their $\mathrm{W}^{ \pm} \rightarrow \mu^{ \pm} v$ decay channel. Their distinctive experimental feature is the presence of a single high- $\mathrm{p}_{\mathrm{T}}$ muon recoiling in the transverse plane to a significant imbalance in the total momentum of the event, which accounts for the undetected neutrino.

Figure 8 (left) shows the muon transverse momentum distribution (black solid circles), measured by ATLAS[14], for events selected online with a low (2$3 \mathrm{GeV} / \mathrm{c}$ ) muon $\mathrm{p}_{\mathrm{T}}^{\mu}$ threshold and fulfilling some quality cuts. An enhancement of events is observed in the region $\mathrm{p}_{\mathrm{T}}^{\mu}>25 \mathrm{GeV} / \mathrm{c}$, where the contribution from muons from $\mathrm{W}$ (and Z) decays is expected. A fit (black solid line) to the data is performed, taking into account two contributions, one for the $\mathrm{W} \rightarrow \mu v$ signal (modelled with a Pythia $\mathrm{pp} \rightarrow \mathrm{W} \rightarrow \mu \nu$ simulation, the normalization left as a free parameter in the fit) (green-hatched histogram) and another for the background (blue-dashed line). The fit describes the data quite well.

CMS [15] has additionally computed the imbalance in the transverse momentum in the event, $\left(p_{\Gamma}\right)$, as the opposite sign of the vectorial sum of all charged particle transverse momenta in the event, with $\mathrm{p}_{\mathrm{T}}>3 \mathrm{GeV} / c$. Events are required to have $p_{\mathrm{T}}^{\mu}>25 \mathrm{GeV} / c$ and $p_{\Gamma}>20 \mathrm{GeV} / c$.

The transverse mass distribution, given by $m_{\mathrm{T}}=$ $\sqrt{2 p_{\mathrm{T}}^{\mu} p_{\Gamma}(1-\cos \phi)}$, being $\phi$ the difference in azimuthal angle between the muon and $p_{\Gamma}$ vectors, for the selected events is presented in Figure 8 (right) for $\mathrm{PbPb}$ (red dots). The data sample of pp collisions collected at the same centre-of-mass energy is analyzed in a similar way, and their $m_{\mathrm{T}}$ distribution is also shown in the figure (blueopen squares). They both represent compatible $\mathrm{W}$ signals, with little background, as they are in good agreement with a W PYTHIA signal simulation, embedded in $\mathrm{PbPb}$ events generated with HYDJET (green-hatched histogram). Residual contamination from other electroweak processes $\left(\mathrm{Z} \rightarrow \mu^{+} \mu^{-}\right.$and $\left.\mathrm{W}^{ \pm} \rightarrow \tau^{ \pm} v\right)(2 \%)$ is subtracted, and the QCD background is estimated to be $\approx 1 \%$ and included as a systematic uncertainty.

From the sample of $\mathrm{W}$ candidates selected in $\mathrm{PbPb}$ and in pp data, normalised with the nuclear factor $\mathrm{T}_{\mathrm{AA}}, \mathrm{CMS}$ computes the $\mathrm{W}$ production cross section in $\mathrm{PbPb}$ collisions per unit of $\mathrm{W}$ rapidity and it is shown in Figure 9 as a function of the number of participants in the collision, $\mathrm{N}_{\text {part }}$. Two main effects are observed: one, the $\mathrm{W}$ production in $\mathrm{PbPb}$ does not depend on the centrality of 

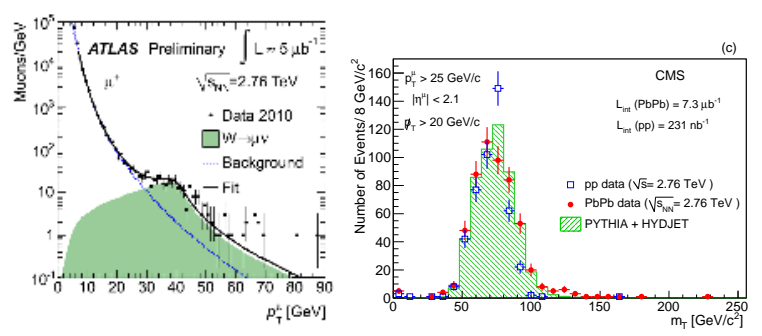

Figure 8. Left: Single-muon transverse-momentum spectrum for $\left|\eta^{\mu}\right|<2.7$ in $\mathrm{PbPb}$ data (black points) measured by ATLAS. $\mathrm{W}^{ \pm} \rightarrow \mu^{ \pm} v$ signal (green-hatched histogram) and background (blue-dashed histogram) contributions are fitted (blacksolid line) to the data. Right: CMS Transverse mass distribution for selected events in $\mathrm{PbPb}$ (red-filled circles) and $\mathrm{pp}$ (blue open squares) data, compared to PYTHIA + HYDJET simulation (hatched histogram). The error bars represent statistical uncertainties.

the collision and the other, the comparison of the individual $\mathrm{W}^{+}$and $\mathrm{W}^{-}$production in $\mathrm{PbPb}$ and $\mathrm{pp}$ data, reflect the different $\mathrm{u}$ and $\mathrm{d}$ quark content in the $\mathrm{Pb}$ nuclei and in the proton. The $\mathrm{R}_{\mathrm{AA}}$ factors obtained are: $\mathrm{R}_{\mathrm{AA}}\left(\mathrm{W}^{+}\right)=$ $0.82 \pm 0.07 \pm 0.09$ and $\mathrm{R}_{\mathrm{AA}}\left(\mathrm{W}^{-}\right)=1.46 \pm 0.14 \pm 0.16$. Nevertheless, the total $\mathrm{W}\left(\mathrm{W}^{+}\right.$plus $\left.\mathrm{W}^{-}\right)$production is consistent, at leading order (LO), with $\mathrm{N}_{\text {coll }}$ scaling, as $\mathrm{R}_{\mathrm{AA}}(\mathrm{W})=1.04 \pm 0.07 \pm 0.12$

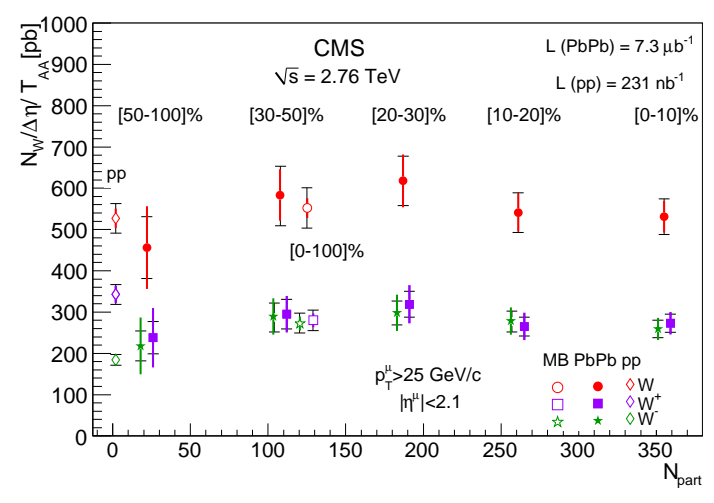

Figure 9. Left: Centrality dependence of normalised $\mathrm{W} \rightarrow \mu \nu$ cross sections in $\mathrm{PbPb}$ collisions, measured by $\mathrm{CMS}$, for all $\mathrm{W}$ candidates (red filled points) and separated by charge, $\mathrm{W}^{+}$ (violet-filled squares) and $\mathrm{W}^{-}$(green-filled stars). The open symbols at $\mathrm{N}_{\text {part }}=120$ represent the minimum bias events. At $\mathrm{N}_{\text {part }}=2$, the corresponding cross sections are displayed for $\mathrm{pp}$ collisions for the same $\sqrt{s}$. The cross sections are given for the phase space region $p_{\mathrm{T}}^{\mu}>25 \mathrm{GeV} / c$ and $\left|\eta^{\mu}\right|<2.1$.

This measurement allows to establish the $\mathrm{W}$ boson as a reference particle, unaffected by the hot and dense medium created in $\mathrm{PbPb}$ collisions.

Whenever the uncertainties (both statistical and systematic) will allow it, the analysis of these bosons will be very useful to constrain predicted nuclear effects (shadowing, energy loss) and give access to quark/antiquark/gluon parton distribution functions (PDF) in the proton and neutron.

\section{Vector Boson plus Jets production}

As seen, data indicate the existance of an energy loss mechanism affecting coloured particles (in the form of jets) while the neutral coloured ones (photons, W and $\mathrm{Z}$ ) are unaffected by the partonic/hadronic medium. Events where both kind of particles are produced (bosons + jets) in the same hard interaction are thus very important to characterise the energy loss mechanism, tagging on the clean bosons and studying the jets distributions.

This has been possible with the data collected during the $2011 \mathrm{PbPb}$ run, using $\gamma / \mathrm{Z}+$ jets selected samples.

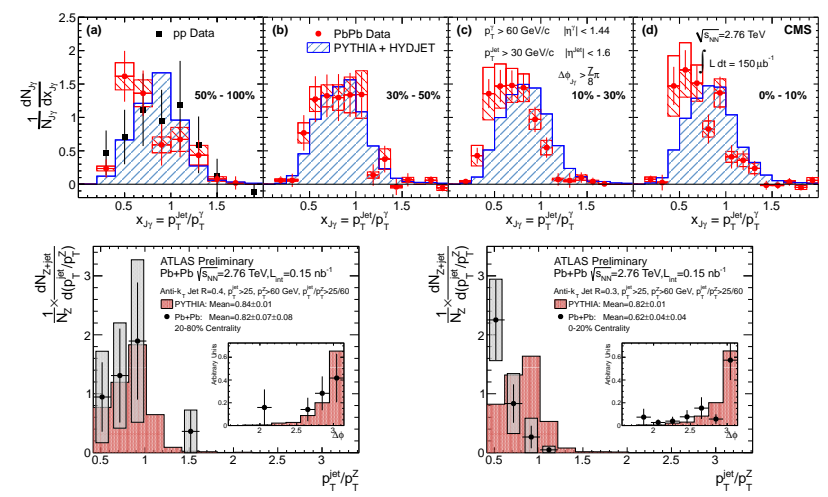

Figure 10. Top: Ratio $\mathrm{p}_{\mathrm{T}}^{\mathrm{J}} / \mathrm{p}_{\mathrm{T}}^{\gamma}$ after background subtraction, by $\mathrm{CMS}$. $\mathrm{PbPb}$ data (filled circles) are compared to pp collisions at $\sqrt{s}=2.76 \mathrm{TeV}$ (filled squares) and to PYHTHIA + HYDJET simulation of the signal (shaded histogram). Four regions in centrality are considered. Bottom: Ratio $\mathrm{p}_{\mathrm{T}}^{\mathrm{J}} / \mathrm{p}_{\mathrm{T}}^{\mathrm{Z}}$, by ATLAS. $\mathrm{PbPb}$ data (filled circles) are compared to signal simulation from PYTHIA. The inset plots show the difference in azimuthal angles between jet and boson.

From the sample of isolated photons and of $\mathrm{Z}$ boson candidates extracted in collision data, and explained in previous sections, those with $\mathrm{p}_{\mathrm{T}}(\gamma / \mathrm{Z})>60 \mathrm{GeV} / c$ and accompanied by a jet with $\mathrm{p}_{\mathrm{T}}(\mathrm{jet})>25-30 \mathrm{GeV}$ at an azimuthal angular separation of the boson sufficient to ensure the jet recoils to it, are selected. The transverse momentum balance between boson and jet is studied by means of the ratio $\mathrm{p}_{\mathrm{T}}^{\mathrm{J}} / \mathrm{p}_{\mathrm{T}}^{\text {boson }}$, which is shown in Figure 10 for the photon + jet sample (as measured by CMS[16]) in the top row of plots and for the Z-boson + jet set (as measured by ATLAS[17]), in the lower plots, for two categories of data according to centrality: peripheral interactions (left) and central collisions (right). Data points from $\mathrm{PbPb}$ collisions are presented, together with the signal simulation from PYTHIA, embedded in $\mathrm{PbPb}$ Hydjet events and additionally with results from pp collisions, in the case of $\gamma+$ jets sample.

There is reasonable agreement among all distributions in the case of peripheral collisions, but a clear $\mathrm{p}_{\mathrm{T}}$ imbalance appears in the case of central collisions. This behaviour can be graphically observed in Figure 11, where 

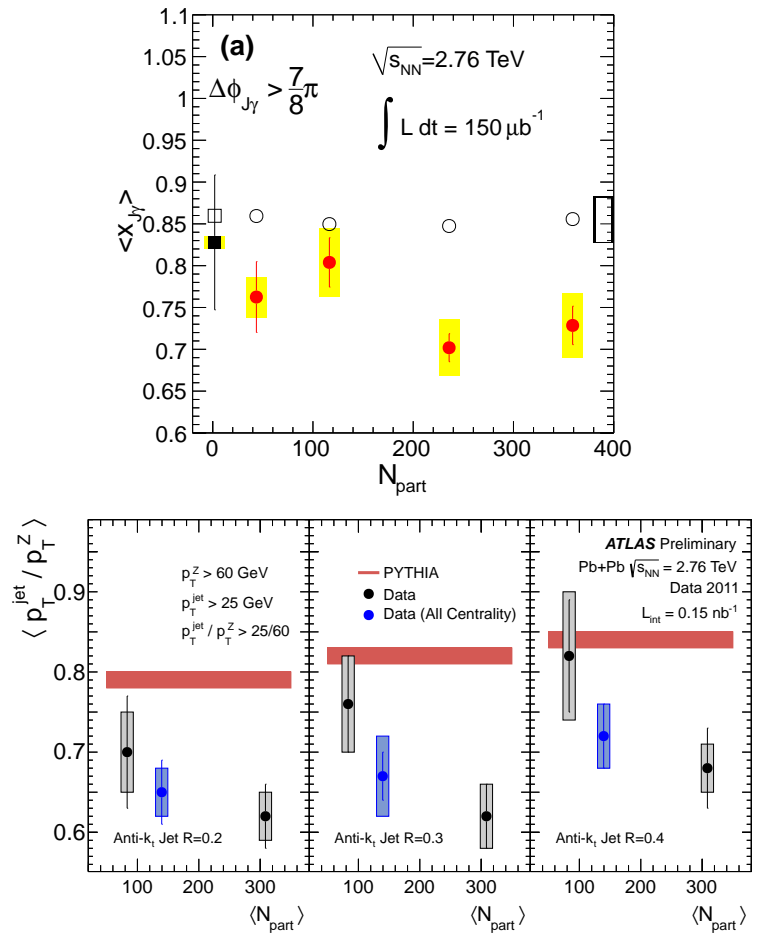

Figure 11. Top: Average ratio of $\mathrm{p}_{\mathrm{T}}^{\mathrm{J}} / \mathrm{p}_{\mathrm{T}}^{\gamma}$ as a function of $\mathrm{N}_{\text {part }}$, by CMS. The same legend from those in Figure 10 top row of plots apply here, being the empty circles the PYTHIA+HYDJET simulation. Bottom: Average ratio $\mathrm{p}_{\mathrm{T}}^{\mathrm{J}} / \mathrm{p}_{\mathrm{T}}^{\mathrm{Z}}$ measured by ATLAS in $\mathrm{PbPb}$ collisions as a function of $\mathrm{N}_{\text {part }}$. The width of the PYTHIA band represents its uncertainty.

the mean value of the previous distributions are shown as a function of the number of participants, $\mathrm{N}_{\text {part }}$.

Similarly, the yield of events with a high- $\mathrm{p}_{\mathrm{T}}$ boson $(\mathrm{Z} / \gamma$ with $\left.\mathrm{p}_{\mathrm{T}}>60 \mathrm{GeV} / c\right)$ and an associated jet $\left(\mathrm{p}_{\mathrm{T}}^{\mathrm{J}}>25-30\right.$ $\mathrm{GeV} / c$ ) relative to all events with a high- $\mathrm{p}_{\mathrm{T}}$ boson, is quantified by means of $\mathrm{N}_{\text {boson+jet }} / \mathrm{N}_{\text {boson }}$, and studied as a function of the collision centrality. A suppression in the pro- duction of boson plus jets events is observed, which quantitatively is in agreement with the nuclear modification factor measured for the inclusive jet yields.

In the future, with statistically more significant $\mathrm{PbPb}$ and pp data samples, at the same centre of mass energies, these studies will allow a more precise insight into the energy loss mechanism, although already today the conjugation of both probes, jets and bosons, in the same event, has rendered important conclusions.

\section{References}

[1] G. Aad et al. ATLAS Collaboration, Phys. Rev. Lett. 105252303 (2010).

[2] S. Chatrchyan et al. CMS Collaboration, Phys. Rev. C 84024906 (2011).

[3] S. Chatrchyan et al. CMS Collaboration, CMS PAS HIN-12-013 and JHEP 10087 (2012).

[4] ATLAS Collaboration, ATLAS CONF-2012-115.

[5] CMS Collaboration, CMS PAS HIN-12-004.

[6] G. Aad et al. ATLAS Collaboration, Phys. Lett. B 719 220 (2013)

[7] ATLAS Collaboration, ATLAS CONF-2012-050.

[8] CMS Collaboration, CMS PAS HIN-12-003.

[9] CMS Collaboration, CMS PAS HIN-13-001.

[10] S. Chatrchyan et al. CMS Collaboration, Phys. Lett. B 710256 (2012).

[11] ATLAS Collaboration, ATLAS CONF-2012-051.

[12] G. Aad et al. ATLAS Collaboration, Phys. Rev. Lett. 110022301 (2013).

[13] CMS Collaboration, CMS PAS HIN-12-008.

[14] ATLAS Collaboration, ATLAS CONF-2011-078.

[15] S. Chatrchyan et al. CMS Collaboration, Phys. Lett. B 71566 (2012).

[16] S. Chatrchyan et al. CMS Collaboration, Phys. Lett. B 718773 (2013).

[17] ATLAS Collaboration, ATLAS CONF-2012-119. 